BULL, AUSTRAL. MATH. SOC.

VOL. 35 (1987) 125-148.

\title{
HOLOAORPHIC CURVES IN THE COMPLEX QUADRIC
}

\author{
Gary R. Jensen, Marco Rigoli and Kichoon Yang
}

A local theory of holomorphic curves in the complex hyperquadric is worked out using the method of moving frames. As a consequence a complete global characterization of totally isotropic curves is obtained.

\section{Introduction.}

Holomorphic curves in a complex quadric axise naturally as the complex conjugate of the Gauss map of a minimal surface in Euclidean space. In addition, such holomorphic curves play a central role in generating a special class of harmonic maps of surfaces into spheres, complex projective space, and the complex Grassmannians. (See Eells-Wood [6], Bryant [2], Chern-Wolfson [5], Ramanathan [14], and many references cited in these papers.)

Received Il March 1986.

$$
\$ A 2.00+0.00 \text {. }
$$

Copyright Clearance Centre, Inc. Serial-fee code: 0004-9729/87 
In this paper we begin a systematic study of the metric differential geometry of holomorphic curves in a quadric by using the method of moving frames on the quadric as a homogeneous space of the orthogonal group. The constructions of this method work for a generic point on a generic curve, but in general a curve will possess certain singular points where the frame construction becomes more complicated. There are three types of singular points: branch points, isotropic points, and real points. The first two types are either isolated or everything, and they present no real problem in the frame construction. However, the real points need not be isolated, and it is not yet clear how to make the frame construction on a neighbourhood of one of these points even if it is isolated. Geometrically the real points are characterised by the property that the osculating space at them has dimension less than two.

We partially characterize the holomorphic curves all of whose points are singular, for each of the three types of singular points. of course if all the points are branch points then the curve is constant. If all the points are real then in Theorem 3.1 it is shown that the curve must be contained in a one dimensional linear section of the quadric. If all of the points are isotropic at every order the curve is called totally isotropic. In Theorems 4.1 and 4.2 we prove by our method the known characterisations of totally isotropic curves in the quadrics of even and odd dimensions, respectively.

\section{Holomorphic curves in the quadric.}

We take as our definition of $Q_{m}$ the non-singular hyperquadric in $\left\langle P^{m+1}\right.$.

$$
Q_{m}=\left\{[z] \in \mathbb{Q P P} \mathbb{P}^{m+1}: t_{z z=0}\right\}
$$

The unitary group $U(m+2)$ acts transitively on $\mathbb{C P}^{m+1}$, and if we choose the point $0={ }^{t}[1,0, \ldots, 0]$ as the origin, then the isotropy subgroup at 0 is $U(1) \times U(m+1)$ and

$$
G P^{m+1}=U(m+2) / U(1) \times U(m+1) \text {. }
$$

We let

$$
\begin{aligned}
& p: U(m+2) \rightarrow 4 P^{m+1} \\
& e \mapsto e \cdot o=\left[e_{1}\right]
\end{aligned}
$$


denote the projection, where $e_{j}$ is the $j^{\text {th }}$ column of $e$.

The orthogonal group $0(m+2)$ is a subgroup of $U(m+2)$ and it maps $Q_{m}$ into itself. If we choose the point

$$
q_{0}=t_{[1, i, 0, \ldots, 0] \in Q_{m}}
$$

as the origin of $Q_{m}$, then the isotropy subgroup of $O(m+2)$ at $q_{0}$ is $S O(2) \times O(m)$ and

$$
Q_{m}=O(m+2) / S O(2) \times O(m)
$$

We let

$$
\begin{aligned}
& q: O(m+2) \rightarrow Q_{m} \\
& e \rightarrow e \cdot q_{0}=\left[e_{1}+i e_{2}\right]
\end{aligned}
$$

denote the projection.

Let $\Omega=\left(\Omega_{b}^{a}\right), \quad 1 \leq a, b, c \leq m+2$, denote the $u(m+2)$ valued Maurer-Cartan form of $U(m+2)$. Then

$$
\Omega_{b}^{a}=-\bar{\Omega}_{a}^{b}
$$

and we have the structure equations

$$
d \Omega_{b}^{a}=-\Omega_{c}^{a} \wedge \Omega_{b}^{c}
$$

The Fubini-study metric $g$ of $\mathbb{C P}^{m+1}$, normalised to have holomorphic sectional curvature equal to four, is characterised by the fact that

$$
p^{*} g=\sum_{2}^{m+2} \Omega_{1}^{a} \bar{\Omega}_{1}^{a} .
$$

We denote the $o(m+2)$ - valued Maurer-Cartan form of $O(m+2)$ by $\theta=\left(\theta_{b}^{a}\right)$. It satisfies

$$
\theta_{b}^{a}=-\theta_{a}^{b}
$$

and the structure equations (2.6). The pull-back of $\Omega$ by the inclusion mapping $O(m+2) \subset U(m+2)$ is $\theta$; in other words $\theta=\Omega \mid O(n+2)$.

Consider the unitary matrix

$$
T=\left|\begin{array}{ccc}
1 / \sqrt{2} & i / \sqrt{2} & 0 \\
i / \sqrt{2} & 1 / \sqrt{2} & \\
& 0 & I
\end{array}\right|
$$


where $I$ denotes the $m \times m$ identity matrix. Then $T \cdot 0=q_{0}$ and consequently

$$
q=p \circ R_{T \mid O(m+2)}
$$

where $R_{T}: U(m+2) \rightarrow U(m+2)$ is right multiplication by $T$. Using the fact that $R_{T}^{*} \Omega=A d\left(T^{-1}\right) * \Omega$, we get from (2.7)

$$
q^{*} g=\sum_{3}^{m+2} \theta^{\alpha} \bar{\theta}^{\alpha}
$$

where we have set

$$
\theta^{\alpha}=\frac{1}{\sqrt{ } 2}\left(\theta_{1}^{\alpha}+i \partial_{2}^{\alpha}\right), \quad 3 \leq \alpha \leq m+2, i=\sqrt{ }-1 .
$$

Thus the pull-back to $Q_{m}$ by any local section of (2.5) of the forms $\theta^{\alpha}$ defines a local unitary coframe in $Q_{m}$ of the Kahler metric induced on it by the Fubini-study metric $g$. This induced metric is given by the pullback of the form (2.9) by the local section.

Let $M$ be a connected Riemann surface and let

$$
f: M \rightarrow Q_{m}
$$

be a non-constant holomorphic map. We define a local orthogonal frome field along $f$ to be a mapping

$$
e: U \subseteq M \rightarrow O(m+2)
$$

such that $q \circ e=f ;$ that is, $f=\left[e_{1}+i e_{2}\right]$.

If we think of $f$ as a holomorphic curve in $\mathbb{C P}^{m+1}$, then $e$ is a local orthogonal frame field along $f$ if and only if $R_{T} \circ e$ is a unitary frome field along $f$ (that is $p \circ R_{T} \circ e=f$, where. $T$ is given by $(2.8)$ ). The situation is illustrated by the diagram

$$
\begin{aligned}
e \nearrow^{O(m+2)} & \subseteq U(m+2) \\
u \subseteq M \rightarrow a_{f} a_{m} & \subseteq \mathbb{P P} m+1
\end{aligned}
$$

Given (2.10) and (2.11), we will write

$$
\theta_{b}^{a}=e^{*} \theta_{b}^{a}
$$


We will use the index conventions

$$
3 \leq \alpha, \beta \leq m+2 \quad ; \quad 1 \leq a, b \leq m+2 .
$$

If $\phi$ is any nowhere zero bidegree $(1,0)$ form in $U$, then the fact that $f$ is holomorphic implies that

$$
\theta_{1}^{\alpha}+i \theta_{2}^{\alpha}=S_{\phi}^{\alpha} \text {, }
$$

for some smooth complex valued functions $S^{\mathbf{\alpha}}$ on $U$.

In fact, if we let

$$
\pi: \mathbb{C}^{m+2} \backslash\{0\} \rightarrow \mathbb{C P} \mathbb{P}^{m+1}
$$

denote the usual projection $z \rightarrow[z]$, then $q \circ e=f$ means that $\pi \circ\left(e_{1}+i e_{2}\right)=f$. From the structure erquations (2.6)

$$
d\left(e_{1}+i e_{2}\right)=i \theta_{2}^{1}\left(e_{1}+i e_{2}\right)+\left(\theta_{1}^{\alpha}+i \theta_{2}^{\alpha}\right) e_{\alpha} \text {, }
$$

and thus

$$
f_{*}=\pi_{*\left(e_{1}+i e_{2}\right)} \circ d\left(e_{1}+i e_{2}\right)=\left(\theta_{1}^{\alpha}+i \theta_{2}^{\alpha}\right) \pi_{*}\left(e_{1}+i e_{2}\right)^{e_{\alpha}},
$$

which verifies (2.15).

We set $S=\left(S^{\alpha}\right) \in \mathbb{C}^{m}$, and observe from (2.18) that $S(x)=0$ for some point $x \in U$ if and only if $f_{*(x)}=0$; that is, $x$ is a bronch point of $f$. If $\tilde{\phi}$ is any other nowhere zero bidegree $(1,0)$ form on $U$, then

$$
\tilde{\phi}=\lambda \phi
$$

for some non-vanishing smooth complex valued function $\lambda$, and by (2.15)

$$
\phi_{1}^{\alpha}+i \theta_{2}^{\alpha}=\lambda^{-1} S^{\alpha} \tilde{\phi}
$$

Thus, setting aside the branch points for the moment, we see that the orthogonal frame $e$ determines a point $[S] \in \mathbb{C P} P^{m-1}$.

Suppose that $\tilde{e}: \tilde{U}+O(m+2)$ is another local orthogonal frame field along $f$, and that $U \cap \tilde{U} \neq \varnothing$. Then on $U \cap \tilde{U}$ we have

$$
\tilde{e}=e K
$$

where $K: U \cap \tilde{U} \rightarrow S O(2) \times O(m)$ is a smooth map given by 


$$
K=\left(\begin{array}{rrr}
\cos t & -\sin t & 0 \\
\sin t & \cos t & \\
& & \\
& 0 & A
\end{array}\right)
$$

where $A: U \cap \tilde{U} \rightarrow O(m)$ is smooth. If we write equations (2.15) for $\tilde{e}$ with the same letters being given a tilde, then

$$
\widetilde{S}=e^{i t} A^{-1} S \text {. }
$$

Consequently, the first step in reducing the bundle $f^{-1} O(m+2) \rightarrow M$ to the first order frames is to determine the orbit structure of the standard action of $O(m)$ on $\mathscr{C} R^{m-1}$. This has been done in HoffmanOsserman [7] (Proposition 2.4, p. 28).

PROPOSITION 2.1. A cross-section of the standard action of $O(m)$ on $\mathbb{C P}^{m-1}$ is (for $m \geq 2$ )

$$
\left\{\left[\begin{array}{c}
1 \\
t i \\
0 \\
\cdot \\
\cdot \\
0
\end{array}\right]: 0 \leq t \leq 1\right\}
$$

That is each orbit of $O(m)$ meets the set (2.23) in exactly one point. The orbits through the points where $0<t<1$ are the principal orbits. The isotropy subgroup of $O(m)$ at these points is $Z_{2} \times O(m-2)$. The other two orbits are singular. The one through the point with $t=1$ is just $Q_{m-2}$ and the isotropy subgroup at this point is $S O(2) \times O(m-2)$. The orbit through the point with $t=0$ is $\mathbb{R} P^{m-1}$ and the isotropy subgroup at this point is $z_{2} \times O(m-1)$.

DEFINITIONS. i) points $[S]$ in the orbit of $t[1, i, 0, \ldots, 0]$ are called isotropic points of $\mathbb{C P}^{m-1}$. If $S$ is given by $(2.15)$ and $[S(x)]$ is isotropic for some $x \in M$, we will call $x$ an isotropic point of $f$. 
The isotropic points are characterised by the condition

$$
t_{S(x)} S(x)=0
$$

By (2.17), $x$ is isotropic if and only if the line through $f(x)$ tangent to $f(M)$ lies in $Q_{m}$.

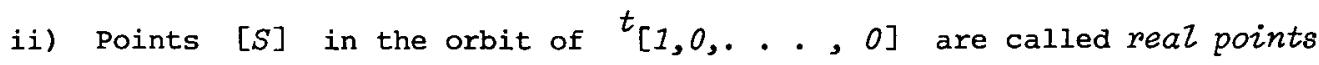
of $a P^{m-1}$. If $[S(x)]$ is real, we will call $x \in M$ a real point of $f$. The real points are characterized by the condition

$$
S(x) \wedge \overline{S(x)}=0 \quad\left(\epsilon \Lambda_{2} \mathbb{C}^{m}\right)
$$

iii) Points of $M$ which are either isotropic, real or branch points will be called singular points of $f$. All other points will be called non-singular.

It is known from the theory of transformation groups (see Jensen [8] that if $x \in M$ is non-singular then there is an open set $U \ni x$ on which there is a smooth orthogonal frame field $e: U \rightarrow O(m+2)$ along $f$ with the property that

$$
[S]: U \rightarrow\left\{{ }^{t}[1, i t, 0, \ldots, 0]: 0<t<1\right\} \subseteq{\varphi^{p^{m-1}}}^{\text {. }}
$$

We will call such a frame field a first order frome field.

Similarly, if every point of $M$ were isotropic (respectively real) then for every point $x \in M$ there would exist an open set $U \ni x$ on which there exists a smooth frame field $e: U \rightarrow O(m+2)$ along $f$ satisfying

$$
[S]={ }^{t}[1, i, 0, \ldots, 0]
$$

(respectively

$$
\left.[S]={ }^{t}[1,0, \ldots, 0]\right) \text {. }
$$

(See remark 2.35 following Corollary 2.4 below for an explanation of why this is possible even if $S(x)=0$ ). We call $f$ first order isotropic (respectively, real) in this case and call $e$ satisfying (2.27) (respectively, (2.28) ) a first order frome field.

However, if $x \in M$ is a singular point which is in the closure of the non-singular points, it is not clear what a first order frame field should be. We shall clarify the situation for branch and isotropic points, but the real points remain a mystery when $m>2$. 
Remark. Let $\phi$ be any nowhere zero bidegree $(1,0)$ form on $U \subseteq M$. Then there is a unique real 1 -form $\omega$ on $U$ satisfying

$$
d \phi=i \omega \wedge \phi \text {. }
$$

In fact, $\omega$ is the Levi-Civita connection form with respect to the unitary coframe field $\phi$ of the Riemannian metric $\phi \bar{\phi}$ on $U$.

PROPOSITION 2.2. Let $e$ be a local orthogonal frome field along $f$. Use the notation of (2.11), (2.13), and (2.15). Then

(2. 30)

$$
\left[d S^{\alpha}+i\left(\omega-\theta_{2}^{1}\right) S^{\alpha}+\theta_{\beta}^{\alpha} S^{\beta}\right] \wedge \phi=0
$$

$$
\left[d\left(^{t} S S\right)+2 i\left(\omega-\theta_{2}^{1}\right){ }^{t} S S\right] \wedge \phi=0,
$$

where $\omega$ is given by (2.29).

Proof. Take the exterior differential of (2.15) and use the structure equations (2.6) and (2.29) to obtain (2.30). Equation (2.31) follows from multiplying $(2.30)$ by $S^{\alpha}$, summing on $\alpha$, and using the fact that $\theta_{\beta}^{\alpha}=-\theta_{\alpha}^{\beta}$.

DEFINITION. Let $U$ be a domain in $M$. A smooth vector-valued function $F: U \rightarrow \mathbb{d}^{n}, n>0$, is of analytic type if for each point $x$ in $U$, if $z$ is a local complex coordinate centered at $x$, then

$$
F=z^{b \tilde{F}}
$$

where $b$ is a positive integer and $\widetilde{F}$ is a smooth $\mathbb{C}$-valued function on a neighbourhood of $x$ such that $\tilde{F}(x) \neq 0$.

It is known that functions of analytic type are precisely the solutions of differential systems

$$
(d F+\psi F) \wedge \phi=0,
$$

where $\Psi$ is a $\mathbb{C}^{n \times n}$-valued 1 -form on $U$ and $\phi$ is a nowhere vanishing bidegree $(1,0)$ form on $U$. If $F$ is a function of analytic type on $U$, then either $F$ is identically zero on $U$ or its zeros are isolated and of finite order (the integer $b$ in (2.32) is the order at $x$ ).

COROLLARY 2.3. Given the notation of Proposition 2.2. Then $S=\left(S^{\alpha}\right): U \rightarrow \mathbb{C}^{m}$ and ${ }^{t} S S: U \rightarrow G$ are of onalytic type. Thus the 
bronch points of $f$ are isolated (of course, as $f$ is holomorphic and assumed non-constant), and either every point of $M$ is an isotropic point or the isotropic points are isolated and of finite order.

COROLLARY 2.4. Given the notation of Proposition 2:2. Let $x_{0} \in U$ be a bronch point. Then the mop $[S]: U \rightarrow \mathbb{C P}^{m-1}$ extends smoothly to $x_{0}$.

Proof. If $z$ is a local complex coordinate centred at $x_{0}$, then $S=z^{b} \tilde{S}$ for some positive integer $b$ and some smooth map $\tilde{S}: V \rightarrow \mathbb{C}^{m}$ such that $\tilde{S}\left(x_{0}\right) \neq 0$, where $x_{0} \in V \subseteq U$. But then $[S(x)]=[\tilde{S}(x)] \in \mathbb{C P} \mathbb{P}^{m-1}$ for $x \neq x_{0}$ and thus $[\tilde{S}]: V \rightarrow \mathbb{C} P^{m-1}$ is the smooth extension to $x_{0}$ of $[S]$.

(2.35) Remark. Suppose that $S$ is not identically zero. By Corollary 2.4: if ${ }^{t} S S \equiv 0$, then $[S]: M \rightarrow Q_{m-2} \subseteq \mathbb{C} P^{m-1}$ is smooth, even at the zeros

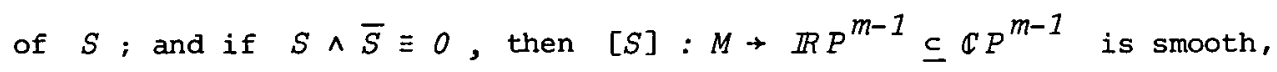
even at the zeros of $S$, where $\mathbb{R} P^{m-1}$ is the $O(m)$-orbit of

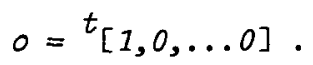

\section{Singular curves.}

We consider now holomorphic curves (2.10) for which every point of $M$ is isotropic, respectively real. We begin with the latter condition, which turns out to be very restrictive.

THEOREM 3.1. Let $f$ be a holomorphic curve (2.10) for which every point of $M$ is real in the sense of (2.25). Then up to $0(m+2)-$ congmence, $f(M)$ is contained in $Q_{1}=\left\{[z] \in Q_{m}: z^{4}=\ldots=z^{m+2}=0\right\}$.

Proof. For any point $x \in M$ there exists a neighbourhood $U$ of $x$ on which there exists a first order frame field $e: U \rightarrow O(m+2)$. By definition (2.28), with respect to such a frame field $S=\left(S^{\alpha}\right)$ $={ }^{t}(P, 0, \ldots, O)$, where $P: U \rightarrow \mathfrak{C}$ is smooth. By (2.15) this means that 


$$
\begin{cases}\text { i) } \theta_{1}^{3}+i \theta_{2}^{3}=P \phi & \\ \text { ii) } \theta_{1}^{\gamma}+i \theta_{2}^{\gamma}=0, & 4 \leq \gamma \leq m+2\end{cases}
$$

Taking the derivative of (3.1) ii) and using (2.6), (3.1) and the fact that $P$ has isolated zeros, we find that

$$
\theta_{3}^{\gamma} \wedge \phi=0, \quad \gamma \geq 4 \text {. }
$$

As $\theta_{3}^{\gamma}$ is real it follows that

$$
\theta_{3}^{\gamma}=0, \quad \gamma \geq 4 \text {. }
$$

Consider the left-invariant, completely integrable distribution on $0(m+2)$

$$
\left\{\theta_{k}^{\gamma}=0: 1 \leq k \leq 3,4 \leq \gamma \leq m+2\right\} \text {. }
$$

It defines the Lie subalgebra $h=o(3) \times o(m-1)$ of $o(m+2)$, and its maximal integral submanifolds are the left cosets of the subgroup $H=O(3) \times O(m-1)$.

Now equations (3.1) and (3.2) say that any first order frame field $e$ is an integral submanifold of (3.3). Thus $e(U) \subseteq A \cdot H$ for some constant $A \in O(m+2)$. As $M$ is connected, this is true for every first order frame field $e$ (that is, $A$ does not depend on $e$ ). As $A^{-1} \cdot e$ is a first order frame field along $A^{-1} \cdot f$, we replace $f$ by the congruent curve $A^{-1} \cdot f$ and assume that $e(U) \subseteq H$ for any first order $e: U \rightarrow O(m+2)$. Hence

$$
f(U)=q \circ e(U) \subseteq q(H)=Q_{1},
$$

where $q$ is the projection (2.5) and $Q_{1} \subseteq Q_{m}$ is defined above in the Theorem. Hence $f(M) \subseteq Q_{1}$.

We turn now to the holomorphic curves for which every point is isotropic. In general a characterisation of these curves awaits an understanding of the non-singular curves. However, for a special class, called the totally isotropic curves, we have a complete characterisation.

Let $f$ be a holomorphic curve (2.10) for which every point is isotropic. We say that $f$ is first order isotropic. We know from section 2 that for any point of $M$ there exists a neighbourhood $U$ containing 
it on which is defined a first order frame field

$$
e: U \rightarrow O(m+2) \text {. }
$$

This means that $[S]={ }^{t}[1, i, 0, \ldots, 0]$, where $S=\left(S^{\alpha}\right)$ is defined by (2.15). Explicitly

$$
\left\{\begin{array}{l}
\text { i) } \theta_{1}^{3}+i \theta_{2}^{3}=s_{1} \phi \\
\text { ii) } \theta_{1}^{4}+i \theta_{2}^{4}=i s_{1} \phi \\
\text { iii) } \theta_{1}^{\gamma}+i \theta_{2}^{\gamma}=0, \gamma \geq 5,
\end{array}\right.
$$

where $\phi$ is a nowhere zero bidegree $(1,0)$ form in $U$, and $s_{1}$ is a smooth complex valued function on $U$ with isolated zeros, each of finite order.

It is convenient to rewrite (3.5) in such a way that a choice of $\phi$ is not made. It is easily seen that (3.5) is equivalent to

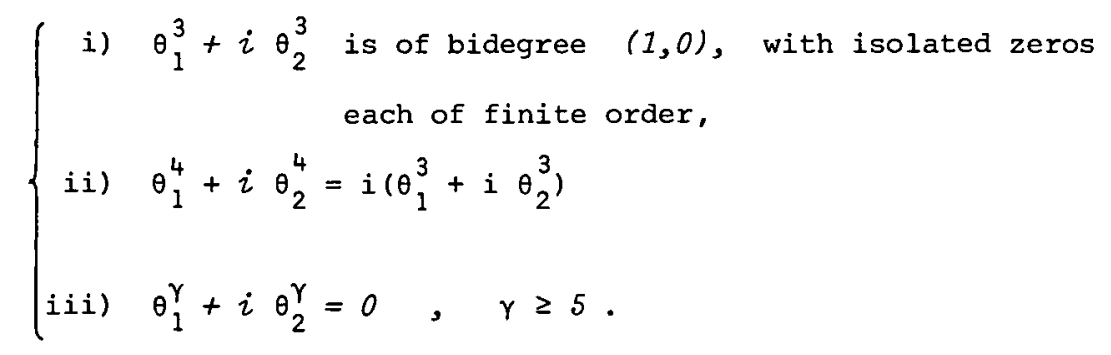

We give now an inductive definition of $f$ being isotropic of order $n$ and of an $n^{\text {th }}$ order frame field along such an $f$, for $n=1$, $2, \ldots,\left[\frac{m}{2}\right]$. Given $n \geq 1$ suppose that $f$ of $(2.10)$ is isotropic of order $n$ and that $e: U \rightarrow O(m+2)$ is a frame field of order $n$ along $f$. Then $e$ is characterised by the conditions

$$
\text { (3.7) }\left\{\begin{array}{l}
\text { ii) } \theta_{2 k-1}^{2 k+2}+i \theta_{2 k}^{2 k+2}=i\left(\theta_{2 k-1}^{2 k+1}+i \theta_{2 k}^{2 k+1}\right) \\
\text { iii) }{ }_{\theta_{2 k-1}{ }_{k}+i \theta_{2 k}+{ }_{2 k}}^{\mu_{k}}=0, \mu_{k} \geq 2 k+3
\end{array}\right.
$$


for $k=1,2, \ldots, n$.

Taking the exterior differential of (3.7) iii) and using the structure equations $(2.6)$ and (3.7) we get that (3.8) $\theta_{2 n+1}^{\mu}+i \theta_{2 n+2}^{\mu}=S_{\phi}^{\mu}, 2 n+3 \leq \mu \leq m+2$, where $\phi$ is any nowhere zero bidegree $(1,0)$ form on $U$, and the $S^{\mu}$ are complex valued smooth functions on $U$. We set

$$
S_{n+1}=\left(S^{\mu}\right): U \rightarrow \mathbb{C}^{m-2 n}
$$

PROPOSITION 3.3. Suppose $f$ is isotropic of order $n$ and let $S_{n+1}$ be given by (3.9). Then:

i) $S_{n+1}$ and $t_{S_{n+1}} S_{n+1}$ are of onalytic type. Up to $0(m+2)$ - congruence:

ii) $f(M) \subseteq Q_{2 n}=\left\{[z] \in Q_{m}: z^{\mu}=0, \mu \geq 2 n+3\right\}$ if and only if $S_{n+1}$ is identically zero;

iii) $f(M) \subseteq Q_{2 n+1}=\left\{[z] \in Q_{m}: z^{\nu}=0, v \geq 2 n+4\right\}$, but not in $Q_{2 n}$, if and only if $S_{n+1} \neq 0$ but $S_{n+1} \wedge \bar{S}_{n+1}$ is identically zero.

Proof. The proof of $i)$ is the same as the proof of Proposition 2.2. The proofs of $i i)$ and iii) follow the same lines as that of Theorem 3.1.

DEFINITION 3.10. $f$ is isotropic of order $n+1$ if it is isotropic of order $n, s_{n+1}$ is not identically zero, and $t_{S_{n+1}} s_{n+1}$ is identically zero.

Remark 3.11. $S_{n+1}$ of (3.9) depends on the choice of $n^{\text {th }}$ order frame field $e$. If $\tilde{e}: \tilde{U} \rightarrow O(m+2)$ is any other $n^{\text {th }}$ order frame field along $f$ (assumed isotropic of order $n$ ), and if $U \cap \tilde{U} \neq \emptyset$, then

$$
\tilde{e}=e K
$$

on $U \cap \tilde{U}$, where $K: U \cap \tilde{U} \rightarrow S O(2)^{n+1} \times O(m-2 n)$ is a smooth map. If we write 


$$
K=\left|\begin{array}{lll}
R\left(t_{1}\right) & & 0 \\
& & R\left(t_{n+1}\right) \\
0 & & A
\end{array}\right|
$$

where

$$
R\left(t_{k}\right)=\left|\begin{array}{cc}
\cos t_{k} & -\sin t_{k} \\
\sin t_{k} & \cos t_{k}
\end{array}\right|, k=1, \ldots, n+1,
$$

and $A: U \cap \tilde{U} \rightarrow O(m-2 n)$ is smooth, then

$$
\tilde{\theta}_{2 k-1}^{2 k+1}+i \tilde{\theta}_{2 k}^{2 k+1}=\exp \left(i\left(t_{k}-t_{k+1}\right)\right)\left(\theta_{2 k-1}^{2 k+1}+i \theta_{2 k}^{2 k+1}\right),
$$

for $k=1, \ldots, n$ and

$$
\widetilde{S}_{n+1}=\exp \left(i t_{n+1}\right) A^{-1} S_{n+1},
$$

where quantities with a tilde refer to $\tilde{e}$.

$$
\text { It follows from (3.14) that } S_{n+1} \text { ( respectively } t_{S_{n+1}} S_{n+1} \text { ), }
$$

vanishes at a point if and only if $\tilde{S}_{n+1}$ (respectively ${ }^{t} \widetilde{S}_{n+1} \widetilde{S}_{n+1}$ ), vanishes at that point. Thus, as was tacitly assumed there, if the conditions on $S_{n+1}$ used in Proposition 3.3 hold for some frame field of order $n$, then they hold for any frame field of order $n$. Furthermore the conditions of definition (3.10) hold for every frame field of order $n$ if they hold for one.

DEFINITION 3.15. Suppose that $f$ is isotropic of order $n+1$. A frame field $e: U \rightarrow O(m+2)$ of order $n+1$ along $f$ is a frame field of order $n$ for which $\left[S_{n+1}\right]=t_{[1, i, 0, \ldots, 0]}$ at every point of $U$.

Remark. It was observed in section 2 that because $S_{n+1}: U \rightarrow c^{m-2 n}$ is of analytic type, if it is not identically zero then the map $\left[S_{n+1}\right]: U \rightarrow \mathbb{C} P^{m-2 n-1}$ is smoothly defined even at the zeros of $s_{n+1}$. That $f$ is isotropic of order $n+1$ is the assumption that 
$\left[S_{n+1}\right](U) \subseteq Q_{m-2 n-2} \subset \mathbb{C} P^{m-2 n-1}$. As the action of $O(m-2 n)$ on $Q_{m-2 n-2}$ defined by (3.14) is the standard action, hence transitive, it follows that for each point of $M$ there exists a neighbourhood on which is defined a smooth frame field of order $n+1$.

DEFINITION 3.16. The holomorphic curve $f$ of $(2.10)$ is totally isotropic if it is isotropic of order $\left[\frac{m}{2}\right]$.

We may interpret Proposition 3.3 as saying in part ii) that $f$ is a totally isotropic curve in $Q_{2 n}$, while in part iii) that $f$ is a totally isotropic curve in $Q_{2 n+1}$.

DEFINITION.3.17. Let $f$ of $(2.10)$ be totally isotropic. A Frenet frome fiezd along $f$ is any frame field of order $\left[\frac{m}{2}\right]$. Frenet frame fields are determined up to changes

$$
\tilde{e}=e K,
$$

where $K$ takes values in

$$
\begin{aligned}
& \text { i) } S O(2)^{n+1} \text { if } m=2 n \text { is even, } \\
& \text { ii) } S O(2)^{n+1} \times O(1) \text { if } m=2 n+1 \text { is odd. }
\end{aligned}
$$

As $O(1)= \pm 1$, we see that in the case $m=2 n+1$ is odd, if we write $e=\left(e_{1}, \ldots, e_{m+2}\right)$ and $\tilde{e}=\left(\tilde{e}_{1}, \ldots, \tilde{e}_{m+2}\right)$, and if (3.18) holds, then $\tilde{e}_{m+2}= \pm e_{m+2}$. Thus the Frenet frames induce a globally defined smooth map

$$
F: M \rightarrow \mathbb{R} P^{m+1}
$$

defined locally by $F=\left[e_{m+2}\right]$. We have the diagram

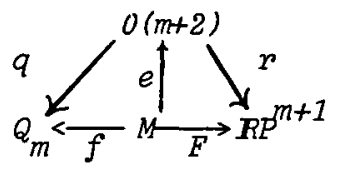

where $e: U \subseteq M+O(m+2)$ is any Frenet frame field and $r$ is the

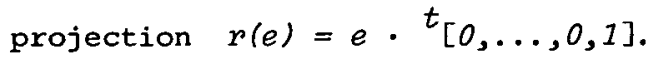


4. The case $m=2 n$.

THEOREM 4.1. Let $f: M \rightarrow Q_{2 n}$ be a holomorphic curve (2.10) which is totally isotropic. Then up to $0(2 n+2)$-congruence $f=h \circ F$, where (4.1)

$$
F: M \rightarrow \mathbb{C} P^{n}
$$

is a linearly full holomorphic curve in $\mathbb{C} P^{n}$, and $h$ is the totally geodesic inclusion of the maximal linear subspace

$$
\begin{gathered}
h: \mathbb{C} P^{n} \rightarrow Q_{2 n} . \\
t_{\left[z_{1}, \ldots, z_{n+1}\right]} \rightarrow{ }^{t}\left[z_{1}, i z_{1}, \ldots, z_{n+1},{ }^{i z} n+1\right.
\end{gathered} .
$$

Conversely, $h \circ F$ is totally isotropic for any linearly full holomorphic curve $F$ of (4.1).

Proof. The idea of the proof is to show that any Frenet field $e$ along $f$ is an integral submanifold of the left-invariant distribution defined on $0(2 n+2)$ by the Lie algebra of the subgroup $U(n+1) \subseteq 0(2 n+2)$. It will follow then that $e$ takes values in a left coset of $U(n+1)$, and as $M$ is connected, we can thus multiply $f$ by an orthogonal transformation and then conclude that any Frenet frame along it takes values in $U(n+1)$. Consequently $f(M) \subseteq q(U(n+1)$ which will be seen to be equal to $h\left(\mathbb{C P} P^{n}\right)$.

For the details we begin with the monomorphism

$$
H: U(n+1) \rightarrow O(2 n+2),
$$

which we define as follows: if $E \in U(n+1)$ has entries $E_{b}^{a}, 1 \leq a, b \leq n+1$, and we write $E_{b}^{a}=A_{b}^{a}+i B_{b}^{a}$ where $A_{b}^{a}$ and $B_{b}^{a}$ are real, then

$$
\left\{\begin{array}{l}
H(E)_{2 b-1}^{2 a-1}=A_{b}^{a}, \quad H(E)_{2 b}^{2 a-1}=B_{b}^{a} \\
H(E)_{2 b-1}^{2 a}=-B_{b}^{a}, \quad H(E)_{2 b}^{2 a}=A_{b}^{a} .
\end{array} .\right.
$$

$H$ is the complex conjugate of the standard inclusion $U(n+1) \subseteq O(2 n+2)$ obtained from the isomorphism 


$$
\begin{aligned}
& R: C^{n+1} \rightarrow R^{2 n+2} \\
& \quad t\left(z^{1}, \ldots, z^{n+1}\right)+\left(x^{1}, y^{1}, \ldots, x^{n+1}, y^{n+1}\right)
\end{aligned}
$$

where $z^{i}=x^{i}+i y^{i}$. That is, for $E \in U(n+1), z \in \mathbb{C}^{n+1}$, $R(E z)=\bar{H}(E) R(z)$, where we have set

$$
\bar{H}(E)=H(\bar{E}) \text {. }
$$

We choose $H$ as we do so that the following diagram commutes:

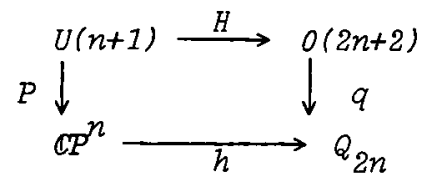

where $p$ and $q$ are the projections (2.2) and (2.5), respectively.

The differential of $H$ gives a Lie algebra monomorphism

$$
H_{*}: u(n+1) \rightarrow o(2 n+2)
$$

which sends the skew-hermitian matrix $E=\left(E_{b}^{a}\right), 1 \leq a, b \leq n+1$, into the skew-symmetric matrix $H_{*} E$ whose components are defined by (4.4), where again $E_{b}^{a}=A_{b}^{a}+i B_{b}^{a}$.

The image $H_{*} u(n+1)$ is thus the Lie subalgebra of $o(2 n+2)$ defined by the left-invariant distribution on $0(2 n+2)$

$$
\theta_{2 b-1}^{2 a}+i \theta_{2 b}^{2 a}=i\left(\theta_{2 b-1}^{2 \alpha-1}+i \theta_{2 b}^{2 a-1}\right), 1 \leq a, b \leq n+1
$$

where $\theta$ is the Maurer-Cartan form of $0(2 n+2)$.

Let $e: U \rightarrow O(2 n+2)$ be any Frenet frame field along $f$. Equations (3.7) show that $e$ is an intergral submanifold of the distribution (4.7). Thus there is a matrix $C \in O(2 n+2)$ such that $e(U) \subseteq C \cdot H(U(n+1))$. If we replace $f$ by the $O(2 n+2)$-congruent curve $C^{-1} \cdot f$, then $C^{-1} \cdot e$ is a Frenet frame field along $C^{-1} \cdot f$, and now $C^{-1} \cdot e(U) \subseteq H(U(n+1))$. Thus we assume that $e(U) \subseteq H(U(n+1))$, which means that (4.8) $f(U)=q \circ e(U) \subseteq q \circ H(U(n+1))=h \circ p(U(n+1))=h\left(\mathbb{C P}{ }^{n+1}\right)$. As $M$ is connected, we have $f(M) \subseteq h\left(\mathbb{O P} P^{n+1}\right)$, and as $h$ is an imbedding there exists a holomorphic curve (4.1) such that $h \circ F=f$. The curve $F$ 
must be linearly full because $f$ cannot lie in a $Q_{k} \subseteq Q_{2 n}, k<2 n$, by Proposition 3.3 .

The mapping (4.2) is totally geodesic because its image is a connected component of the fixed point set of the isometry of $Q_{2 n}$ defined by $V \in O(2 n+2)$,

$$
V=\left|\begin{array}{lll}
J & & 0 \\
0 & \cdot & J
\end{array}\right|
$$

where $J=\left|\begin{array}{rr}0 & -1 \\ 1 & 0\end{array}\right|$, (see Kobayashi [1], p. 59). In fact, the fixed point set of $V$ is

$$
q \circ H(U(n+1)) \cup q \circ \bar{H}(U(n+1)),
$$

where $\bar{H}$ was defined in (4.5). These two sets, the first of which is $h\left(\mathbb{C P} P^{n}\right)$, are connected and disjoint.

To prove the converse of the Theorem, let the linearly full holomorphic curve $F$ of (4.1) be given. To show that the holomorphic curve $f=h \circ F$ is totally isotropic it suffices to show that $M$ is covered by open sets $U$ on each of which there is a smooth frame field $e: U \rightarrow O(2 n+2)$ along $f$, which satisfies the equations (3.7) for $k=n$. To do this we let $E: U \rightarrow U(n+1)$ be a Frenet frame field along $F$. (see Jensen-Rigoli [3] for details.) This means that

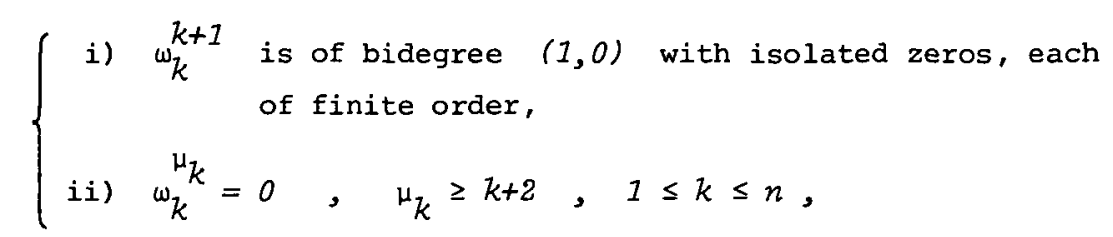

where $\omega_{b}^{a}=E^{*} \Omega_{b}^{a}, 1 \leq a, b \leq n+1$, and $\Omega=\left(\Omega_{b}^{a}\right)$ is the MaurerCartan form of $U(n+1)$.

We have the commuting diagram

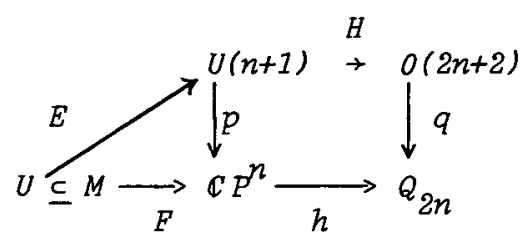


which shows that $e=H \circ E$ is an orthogonal frame field along $f=h \circ F$. Furthermore, as

$$
H^{*} \Theta=H_{*} \circ \Omega,
$$

we have

$$
\theta=e^{*} \theta=E^{*}\left(H_{*} \circ \Omega\right)=H_{*} \circ \omega,
$$

where $\omega=E^{*} \Omega$. From $(4.13,(4.10)$ and (4.4) it follows that the entries of $\theta$ satisfy

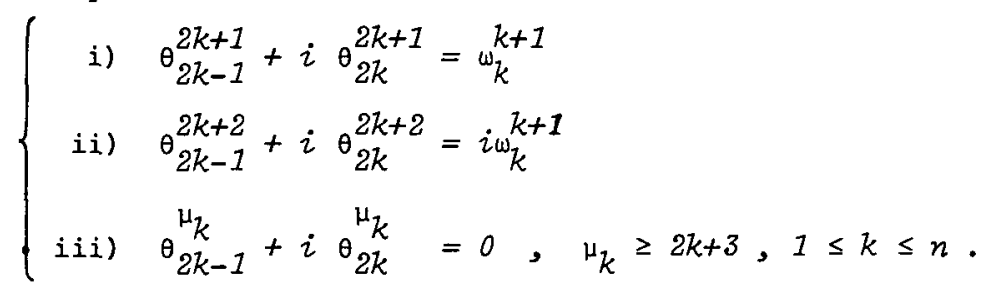

By (4.10) i) and (4.14) $e^{*} \theta$ satisfies equations (3.7), and thus $f$ is totally isotropic and $e$ is a Frenet frame field along it. This completes the proof of Theorem 4.1.

COROLLARY 4.2. Let $M d s^{2}$ be a Riemannian surface. If $F: M \rightarrow \llbracket P^{n+1}$ is harmonic, then $h \circ F: M \rightarrow Q_{2 n}$ is harmonic.

Proof. The composition $h \circ F$ of a harmonic map $F$ with a totally geodesic map $h$ is always harmonic.

Our Theorem 4.1 should be compared to the theorem of Lawson ([1], pp. 165-166). If

$$
X: M \rightarrow \mathbb{R}^{2 n+2}
$$

is a minimal immersion, and $f: M+Q_{2 n}$ is its Gauss map (defined so that it is holomorphic, not anti-holomorphic), then we have a diagram

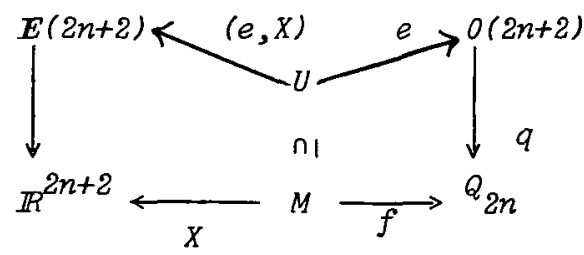

where $\boldsymbol{E}(2 n+2)=0(2 n+2) \cdot \mathbb{R}^{2 n+2}$ is the group of rigid motions, and $(e, X): U \rightarrow \mathbb{E}(2 n+2)$ denotes a Darboux frame field along $X$. This means 
precisely that $e: U \rightarrow O(2 n+2)$ is an orthogonal frame field along $f$.

There exists an orthogonal complex structure on $\mathbb{R}^{2 n+2}$ with respect to which $X$ is holomorphic if and only if about any point of $M$ there exists a frame field $(e, X)$ which takes values in a fixed left coset of the subgroup $H(U(n+1)) \cdot \mathbb{R}^{2 n+2}$. This turns out to be precisely the condition that $e$ satisfy equations (3.7), that is that $f$ is totally isotropic.

\section{The case $m=2 n+1$.}

THEOREM 5.1. Let $f: M \rightarrow Q_{2 n+1}$ be a holomorphic curve (2.10) which is totally isotropic. Then the mop

$$
F: M \rightarrow \mathbb{R P}^{2 n+2}
$$

defined in (3.20) is linearly full and pseudoholomorphic (possibly branched) and the directrix curve of $F$ is $\bar{f}$. Conversely, if (5.1) is any linearly full, bronched pseudoholomorphic curve, then the complex conjugate of its directrix curve is a totally isotropic curve in ${ }_{2 n+1}$.

We will define pseudoholomorphic and directrix curve, and give references, in the course of the proof.

Proof. In Jensen-Rigoli [9] isometric minimal immersions $F: M \rightarrow S^{2 n+2}$ were studied which had the additional property of being "isotropic of order $n$ ". Following Calabi [3], we now use the term pseudoholomorphic for these maps. If the metric on $M$ is changed conformally, then $F$ is no longer isometric minimal, but is harmonic. Referring to (24) in Jensen-Rigoli [9], we define $F$ to be a linearly full branched pseudoholomorphic map if for each point of the Riemann surface $M$ there exists a neighbourhood $U$ on which there is a frame field

$$
E: U \rightarrow O(2 n+3)
$$

along $F$ (meaning that $F=\left[E_{1}\right]$ ) such that 
$(5.3)$

$$
\left\{\begin{array}{l}
\text { i) } \phi_{1}^{2}+i \phi_{1}^{3}, \\
\text { ii) } \phi_{3}^{5}+i \phi_{3}^{4}=i\left(\phi_{2}^{5}+i \phi_{2}^{4}\right), \\
\text { iii) } \phi_{2 j-1}^{2 j+1}+i \phi_{2 j-1}^{2 j}=-i\left(\phi_{2 j-2}^{2 j+1}+i \phi_{2 j-2}^{2 j}\right),
\end{array}\right.
$$

are all of bidegree $(1,0)$ with isolated zeros, each of finite order; and

$$
\text { (5.4) }\left\{\begin{array}{cl}
\text { i) } \phi_{1}^{k}=0 \quad, \quad k \geq 4 \\
\text { ii) } \phi_{2}^{k}=0=\phi_{3}^{k}, k \geq 6 \\
\text { iii) } \phi_{2 j-2}^{k}=0=\phi_{2 j-1}^{k}, \quad k \geq 2 j+2
\end{array}\right.
$$

where $j=3, \ldots, n+1$, and $\left(\phi_{b}^{a}\right)=E^{*}\left(\theta_{b}^{a}\right)$ is the pull back of the Mauxer-Cartan form of $0(2 n+3)$. Such a frame field is called a Frenet frame field along $F$.

The significance of the notion of pseudoholomorphic map is that if $M$ is the Riemann sphere and $F$ is a conformal harmonic mapping, then $F$ is necessarily pseudoholomorphic.

Let $f: M \rightarrow Q_{2 n+1}$ of $(2.10)$ be totally isotropic, and let $e: U \rightarrow O(2 n+3)$ be a Frenet frame field along $f$. Let $\varepsilon_{1}, \varepsilon_{2}, \ldots$, $\varepsilon_{2 n+3}$ denote the standard basis of $\mathbb{R}^{2 n+3}$ and let

$$
G=\left(\varepsilon_{2 n+3}, \varepsilon_{2 n+1}, \varepsilon_{2 n+2}, \varepsilon_{2 n}, \varepsilon_{2 n-1}, \ldots, \varepsilon_{2}, \xi^{\prime}\right) .
$$

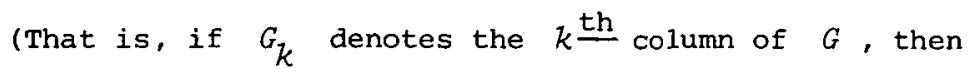

$\left.G_{1}=\varepsilon_{2 n+3}, G_{2}=\varepsilon_{2 n+1}, G_{3}=\varepsilon_{2 n+2}, G_{j+3}=\varepsilon_{2 n+1-j}, 1 \leq j \leq 2 n\right)$.

Then $G \in O(2 n+3)$ and by $(3.20)$

$$
E=e G: U \rightarrow O(2 n+3)
$$

is an orthogonal frame field along $F$. Making the calculation

$$
\phi=E^{*} \theta=G^{-1} e^{*} \theta G
$$

and using (3.7) we obtain equations (5.4), and 
$(5.6)$

$$
\left\{\begin{array}{l}
\text { i) } \phi_{1}^{2}+i \phi_{1}^{3}=\theta_{2 n+3}^{2 n+1}+i \theta_{2 n+3}^{2 n+2} \\
\text { ii) } \phi_{2}^{5}+i \phi_{2}^{4}=\theta_{2 n+1}^{2 n-1}+i \theta_{2 n+1}^{2 n} \\
\text { iii) } \phi_{3}^{5}+i \phi_{3}^{4}=\theta_{2 n+2}^{2 n-1}+i \theta_{2 n+2}^{2 n} \\
\text { iv) } \phi_{2 j+2}^{2 j+5}+i \phi_{2 j+2}^{2 j+4}=\theta_{2(n-j)+2}^{2(n-j)-1}+i \theta_{2(n-j)+2}^{2(n-j)} \\
\text { v) } \phi_{2 j+3}^{2 j+5}+i \phi_{2 j+3}^{2 j+4}=\theta_{2(n-j)+1}^{2(n-j)-1}+i \theta_{2(n-j)+1}^{2(n-j)} \\
j=1,2, \ldots, n-1,
\end{array}\right.
$$

and

$$
\begin{cases}\text { i) } \phi_{2}^{k}=0=\phi_{3}^{k}, & 6 \leq k \leq 2 n+3 \\ \text { ii) } \phi_{2 j+2}^{k}=0=\phi_{2 j+3}^{k}, & 2 j+6 \leq k \leq 2 n+3, j=1, \ldots, n-1 .\end{cases}
$$

Equations (5.6) and (5.7) combined with (3.7) show that $E$ satisfies equations (5.3) and (5.4). Hence $F$ is a linearly full branched pseudoholomorphic map and $E$ is a Frenet frame field along it.

The directrix curve of a pseudoholomorphic curve in $\mathbb{R} P^{2 n+2}$ (or $S^{2 n+2}$, was introduced in Calabi [3] and Chern [4], and was studied further by Barbosa [1]. In Jensen-Rigoli [10] the directrix curve is described locally in terms of any Frenet frame field $E: U \rightarrow O(2 n+3)$ along $F$. Writing $E=\left(E_{1}, \ldots, E_{2 n+3}\right)$, then its directrix is the map

$$
\bar{f}: M \rightarrow Q_{2 n+1}
$$

defined on $U$ by $\bar{f}=\left[E_{2 n+2}+i E_{2 n+3}\right]$. This map is globally defined because any other Frenet frame field $\widetilde{E}: \widetilde{U} \rightarrow 0(2 n+3)$ satisfies on $U \cap \tilde{U}$ (assumed non-empty)

$$
\tilde{E}=E K
$$

where $K: U \cap \tilde{U} \rightarrow O(1) \times S O(2)^{n+1}$.

In Jensen-Rigoli [10] it was observed that each of the maps $\left[E_{2 j}+i E_{2 j+1}\right], j=1, \ldots, n+1$ is globally defined (by (5.9)) from $M$ 
into $Q_{2 n+1}$ and is harmonic. The directrix curve $(j=n+1)$ is antiholomorphic.

Given a totally isotropic map $f: M \rightarrow Q_{2 n+1}$ with a local Frenet frame $e: U \rightarrow O(2 n+3)$, then $E$ of (5.5) is a Frenet frame along the pseudoholomorphic map $F$ of (5.1). Its directrix is defined locally by

$$
\left[E_{2 n+2}+i E_{2 n+3}\right]=\left[e_{2}+i e_{1}\right],
$$

by (5.5), and this latter map is $\left[e_{1}-i e_{2}\right]=\bar{f}$ by (2.11).

Conversely, given a linearly full branched pseudoholomorphic map (5.1), let $E$ of (5.2) be a Frenet frame field along it. As mentioned above, its directrix $\bar{f}=\left[E_{2 n+2}+i E_{2 n+3}\right]$ is antiholomorphic. If $e=E G^{-1}$, where $G$ is given in (5.5), then a straight forward computation shows that $e$ is an orthogonal frame field along the holomorphic curve $f=\overline{\bar{f}}: M \rightarrow Q_{2 n+1}$ and satisfies equations (3.7) as a result of $E$ satisfying (5.3) and (5.4). Hence $f$ is totally isotropic.l

These constructions and correspondences are summarised in the following commuting diagram

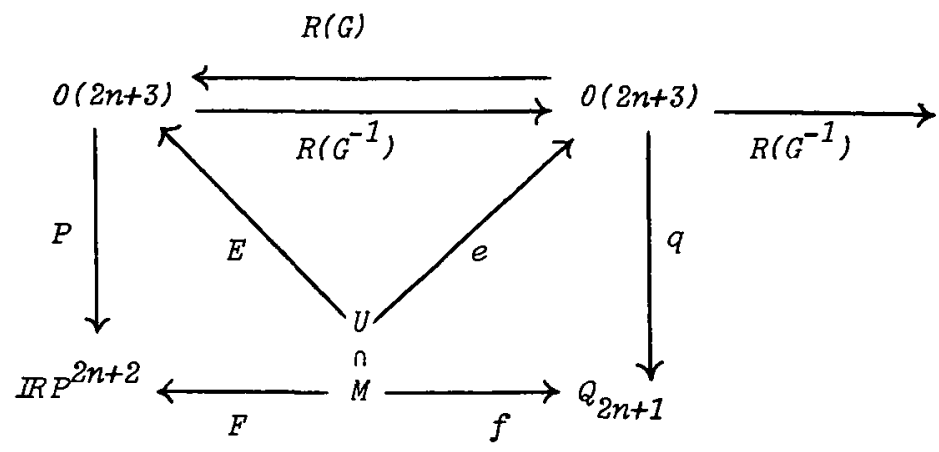

where $R(G)$ (respectively $R\left(G^{-1}\right.$ ) is right multiplication by $G$ (respectively $G^{-1}$ ) of $(5.5)$, and $p$ is the projection map (2.2) restricted to $0(2 n+3)$.

\section{References}

[1] K.J. Barbosa, "On minimal immersions of $S^{2}$ into $S^{2 m}$." Trans. Amer. Math. Soc. 210 (1975), 75-106. 
[2] R.L. Bryant, "Conformal and minimal immersions of compact surfaces into the 4-sphere", J. Differential Geom. 17 (1982), 455-473.

[3] E. Calabi, "Quelques applications de l'analyse complexe aux surfaces d'aire minime", Topics in complex manifolds, univ. of Montreal, Montreal, Canada, 1968, 59-81.

[4] S.S. Chern, "On minimal spheres in the four sphere", Selected Popers, Springer Verlag, New York, 1978, 421-434.

[5] S.S. Chern and J. Wolfson, "Harmonic maps of $S^{2}$ into a complex Grassmann manifold", Proc. Nat. Acad. Sci. U.S.A., 82 (1985), 2217-2219.

[6] J. Eells and J. Wood, "Harmonic maps from surfaces to complex projective spaces", Ad. in Math. 49 (1983), 217-263.

[7] D.A. Hoffman and R. Osserman, "The geometry of the generalized Gauss map", Mem. Amec.Math.Soc. 28 (no. 236) (1980)

[8] G.R. Jensen, "Higher order contact of submanifolds of homogeneous spaces", Lecture Notes in Math. Vol. 610, Springer, Berlin,1977.

[9] G.R. Jensen and M. Rigoli, "Minimal surfaces in spheres", Special Volume, Rend. Sem. Math. Fis., Torino, (1983), 75-98.

[10] G.R. Jensen and M. Rigoli, "A class of harmonic maps from surfaces into real Grassmanians", Special Volume, Rend. Sem. Mat. Fis., Torino, (1983), 99-116.

[11] G.R. Jensen and M. Rigoli, "Quantization of curvature of harmonic two spheres in complex projective space" (preprint).

[12] s. Kobayashi, Transformation Groups in Differential Geometry, Springer-Verlay, New York, 1972.

[13] H.B. Lawson, Lectures on Minimal Submonifolds, Vol.1, Publish or Perish, Berkeley, 1980.

[14] J. Ramanathan, "Haxnonic maps from $S^{2}$ to $G(2,4) "$. J. Differential Geom. 19 (1984), 207-219.

[15] K. Yang, "Frenet formulae for holomorphic curves in the two quadric", BuzZ.AustraZ.Math.Soc. 33 (1986), 195-206.

Department of Mathematics,

Box 1146

Washington University

SAINT LOUIS

Missouri 63130

United States of America 
International Center for Theoretical Physics

Strada Costiera II

MIRAMARE TRIESTE

Italy

Department of Mathematics

Arkansas State University

State University

Arkansas 72467

United States of America. 